\title{
Distributed parameter model simulation tool for PEM fuel cells
}

\author{
Maria Sarmiento-Carnevali ${ }^{\mathrm{a}, 1}$, Maria Serra ${ }^{\mathrm{a}}$, Carles Batlle ${ }^{\mathrm{b}}$ \\ ${ }^{a}$ Institut de Robòtica i Informàtica Industrial (CSIC-UPC), C/ Llorens i Artigas 4-6, \\ 08028 Barcelona \\ ${ }^{b}$ Departament de Matemàtica Aplicada IV \& IOC, Universitat Politècnica de Catalunya, \\ EPSEVG, Av. V. Balaguer s/n, 08800 Vilanova i la Geltrú
}

\begin{abstract}
In this work, a simulation tool for proton exchange membrane fuel cells (PEMFC) has been developed, based on a distributed parameter model. The tool is designed to perform studies of time and space variations in the direction of the gas channels. Results for steady-state and dynamic simulations for a single cell of one channel are presented and analyzed. Considered variables are concentrations of reactants, pressures, temperatures, humidification, membrane water content, current, among others that have significant effects on the performance and durability of PEMFC.
\end{abstract}

Keywords: PEMFC, distributed parameter modeling, dynamic simulation

\section{Introduction}

The proton exchange membrane fuel cells (PEMFC) technology has been incorporated to a wide range of portable, stationary and automotive applications in recent years [1]. However, despite current developments, PEMFC

\footnotetext{
${ }^{1}$ Corresponding author. Tel.: +34 (93) 40158 05; fax: +34 (93) 40157 50. E-mail address: m.sarmiento@iri.upc.edu (M.L. Sarmiento-Carnevali).
} 
are still not accepted as a practical power generator. The key challenge is to reduce the cost and achieve a high performance and long life of the cells.

Along-the-channel variations of important variables such as concentrations of hydrogen, oxygen and water, as well as temperature, have significant effects on the performance and durability of PEMFC. All these variables exhibit spatial dependence in the direction of the channels of the anode and cathode and, therefore, it is necessary to introduce partial differential equations (PDE) to accurately model such variations.

Distributed parameter first principles modeling allows, indeed, the detailed study of the fundamental phenomena that occur within a PEMFC. Bernardi and Verbrugge [2] and Springer et al. [3] developed pioneering distributed parameter models. These models, both one-dimensional, analyzed species transport, water addition and removal, cathode flooding and the effect of gas humidification. Later, Rowe and Li [4] developed a one-dimensional, non-isothermal model of a PEM fuel cell, incorporating water and temperature distribution to investigate the operating conditions effects on the cell performance, thermal response and water management.

Methekar [5] developed a two-dimensional, along-the-channel, distributed parameter model to perform dynamic analysis and design linear control strategies for PEMFC. A detailed two-dimensional simulation model was presented by Mangold et al. [6] for control purposes. Um et al. [7] developed a 2D model to simulate proton exchange membrane fuel cells. The model accounts simultaneously for electrochemical kinetics, current distribution, hydrodynamics, and multicomponent transport. A single set of conservation equations valid for flow channels, gas diffusion electrodes, catalyst layers, 
and the membrane region are presented. Kulikovsky [8] published a semianalytical model of PEMFC, which takes into account oxygen and water transport across the cell and along the channel. The two-dimensional models can be divided into two categories. One group of models describes the plane perpendicular to the flow channels, while the other group of models describes the direction across the fuel cell and the direction along the flow channel. Each group has its advantages and drawbacks. In the first group of models, the effect of flow channel dimension and configuration can be studied, however, changes in the temperature and reactants fraction cannot be accounted for. The second group of models can predict the temperature and concentration profiles along the direction of the flow channel, but cannot simulate the effect of flow channel and rib size.

Three-dimensional models have been developed by various research groups $[3,9]$. Schumacher et al. [10] presented a 2D $+1 \mathrm{D}$ to take the in-plane and the through-plane dimensions of fuel cells into account. The anode and cathode are included as 2D domains, and a nonlinear 1D model represents the membrane electrode assembly (MEA). Tao [11] developed an important three-dimensional, two-phase, non-isothermal unit cell model in order to perform parameter sensitivity examination. Um and Wang [12] developed a computational fuel cell dynamics (CFCD) model to study three-dimensional (3D) interactions between mass transport and electrochemical kinetics in PEMFC. In addition to different geometrical assumptions, important multiscale modeling approaches have been published $[13,14,15]$. Multiscale models allow the study of system behavior at different levels (from smaller to larger scales). On each level, particular approaches are used for description of the system. 
Following levels are usually distinguished: quantum mechanical models (information about electrons is included), level of molecular dynamics models (information about individual atoms is included), mesoscale or nano level (information about groups of atoms and molecules is included), level of continuum models, level of device models, among others.

Moreover, there are various simulation tools for PEMFC [16], but very few are flexible enough to manipulate the underlying model equations. This work is focused on the development of a simulation tool for two intended purposes: (1) the analysis of variables with important spatial variations along the channel, and (2) the application of model order reduction techniques to design distributed parameter model-based controllers, aimed at the control of these spatial variations [17].

In this work, the implemented tool is based on a distributed parameter model $(1+1 \mathrm{D})$ to be used in model-based controllers. This type of 2D models facilitates the detailed study of along-the-channel spatial variations of important variables regarding performance and durability of PEMFC, as well as a simplified analysis of variables in the direction across the MEA (important for water transport studies), by approximating spatial gradients. Therefore, the emphasis on channel direction profiles makes the model suitable for control design that takes into account spatial profiles, and simple enough (not being a 1D model) to apply model order reduction techniques that are very complex. These features make the model an appropriate choice over the wide range of geometrical assumptions, such as $2 \mathrm{D}, 2 \mathrm{D}+1 \mathrm{D}, 3 \mathrm{D}$ (previously mentioned and referenced).

The paper is organized as follows. Section 2 shows the structure of the 
modeled system. Section 3 describes the model equations. Section 4 covers the tasks of simulation tool development (discretization and implementation). Section 5 presents simulation results and Section 6 concludes on the work developed.

\section{Description of the system}

This work considers a single cell of one channel (Fig. 1) that includes all the functional parts of the PEMFC: bipolar plates (BP), gas channels (GC), gas diffusion layers (GDL), catalyst electrode layers (CL), a proton exchange membrane and a cooling system. This simple model suits the detailed analysis of spatial variations. The case study selected in the work is a $0.4 \mathrm{~m}$ along-the-channel single cell (area 0.4 x 10-3 m) with Nafion 117 membrane. The parameters of the membrane are taken from $[18,19]$.

\section{Distributed parameter model}

The model equations are based on the work by Mangold et al. [6]. This is not only a recent model, but it is also one of the most detailed set of PEMFC equations in the open literature. The model is $1+1 \mathrm{D}$ with approximation of gradients by a fixed number of points in y-direction and partial differential equations (PDE) along the channel (z-direction) (Fig. 1).

Physical phenomena occurring within the gas channels is represented by the solution of conservative equations for mass, momentum and energy. The principle of mass conservation is used to model reactant concentrations. A pressure drop relation, derived from simplified Navier-Stokes equations, allows the calculation of the flow velocity. The ideal gas law is used to calculate 


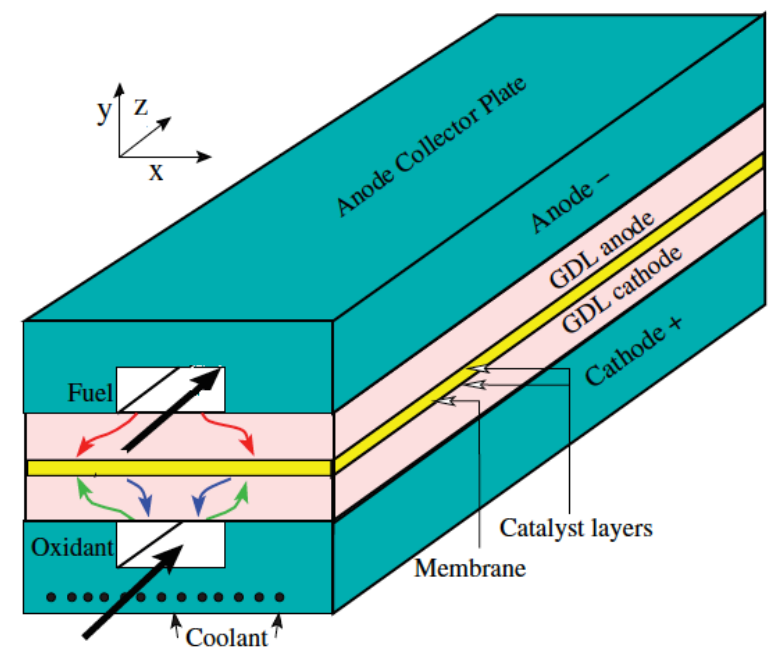

Figure 1: Structure of the fuel cell considered in this work

flow pressure. An energy balance is used to determine the flow temperature.

Stefan-Maxwell diffusion equations for a multicomponent gas are used to define the mole fraction gradient in the gas diffusion layers (GDL). The reaction rates are modeled by Butler-Volmer equations and Faraday's law. Membrane behavior is described by the calculation of membrane water content, water concentration, drag coefficient, net water flux and protonic conductivity. The gas diffusion layers (GDL), catalyst layers (CL) and membrane are considered to be at the same temperature level. These PEMFC components form the solid part (SP).

Liquid water formation is not considered. The model validity is restricted to one-phase conditions, where condensation in gas channel and gas diffusion layers is negligible. Considering liquid water formation is a future objective but, in this moment, using the simulation tool for control purposes by means 
of model order reduction techniques is a priority.

An energy balance for the solid part, similar to the energy balances of the gas channels (GC), is used to calculate the solid part temperature. Current transport is described by governing equations for conservation of charge and the cell potential is calculated by the polarization curve equation. In this relation, activation polarization losses, ohmic losses and concentration losses are considered. The combination of these laws results in a set of PDE and algebraic constraints.

In this work, important effort on presenting membrane model details and calculations has been made. In addition, the entire spatial discretization process of equations describing PEMFC phenomena is presented. Moreover, a widely used software (Matlabß) was selected to develop the simulation tool. Finally, interesting and different results are presented: along-the-channel behavior and time evolution of variables at specific channel points. Actually, there is special emphasis on spatial and temporal variance analysis, given its importance for performance and durability of PEMFC.

\section{Model implementation}

The set of PDE and algebraic constraints was discretized spatially along the channel using finite differences [20]. As a result, a set of differential algebraic equations (DAE) was obtained [21]. This new set of model equations was implemented and numerically solved in Matlabß, using a DAE solver.

\subsection{Model discretization}

Partial differential equations and algebraic constraints have been discretized by finite differences into MZ points (uniformly distributed) along 
z-direction, starting from $\mathrm{z}=0 \mathrm{~m}$ (first point) until $\mathrm{z}=0.4 \mathrm{~m}$ (channel length). Along y-direction, only a few points are considered, depending on the submodel of interest. For variables such as different temperatures levels, no y-direction variation is considered (gas channels temperatures, for instance). However, for variables such as water fluxes or molar fractions, different points along y-direction are studied (middle point of channel, middle point of membrane, membrane boundary to anode side, membrane boundary to cathode side, among others). In the following sections, all the discretized equations and their purpose within the model are presented ( $k$ is an index that denotes mesh points).

\subsubsection{Gas channels submodel}

The gas channel equations allow the calculation of concentration of gases, flow velocities, flow pressure and temperature in the gas channels.

- Mass balances equations

The general equation for mass conservation is discretized as follows:

$$
\frac{d c_{i, k}^{j}}{d t}=-\frac{v_{k}^{j} c_{i, k}^{j}-v_{k-1}^{j} c_{i, k-1}^{j}}{\Delta z}-\frac{\dot{n}_{i, k}^{j}}{\delta^{j}}
$$

From these equations, the values of the concentrations $c_{i, k}^{j}$ at each mesh point, but the first, are determined. The boundary condition remains an algebraic equation as follows:

$$
\left.v_{0}^{j} c_{i, 0}^{j}\right|_{0, t}=\dot{n}_{i, i n}^{j}
$$


This algebraic equation is used to calculate concentrations at the beginning of the gas channels.

The superscript $j$ is used to denote anode side $(A)$ or cathode side $(C)$. The subscript $i$ indicates the species index. On anode side, it can be either $\mathrm{H}_{2}$ or $\mathrm{H}_{2} \mathrm{O}$. The water component might be present in the case of hydrogen humidification [20]. On cathode side, $i$ can be either $\mathrm{O}_{2}$, or $\mathrm{N}_{2}$ or $\mathrm{H}_{2} \mathrm{O}, \delta^{j}$ is gas channel thickness in y-direction (Fig. 1), $\dot{n}_{i}^{j}$ denote molar flow densities between gas channels and gas diffusion layers, $\dot{n}_{i, i n}^{j}$ denote inlet molar flow densities (inlet flow divided by cross-sectional area of the gas channels [6])

- Flow velocity equations

Normally, velocity vectors for flow dynamics are determined by the conservation of momentum equations, the so-called Navier-Stokes equations [22]. Considering a set of assumptions, such as neglecting the acceleration terms, the Navier-Stokes equation can be simplified into a pressure drop relation similar to Darcy's law [22]. Calculation of flow velocities for both anode gas channel and cathode gas channel, using forward differencing is:

$$
v_{k}^{j}=-K^{j} \frac{p_{k+1}^{j}-p_{k}^{j}}{\Delta z}
$$

Considering boundary condition ( $M Z$ is the number of mesh points):

$$
v_{M Z}^{j}=-K^{j} \frac{p^{a m b}-p_{M Z-1}^{j}}{\Delta z}
$$

- Flow pressure equations

Ideal gas law is used to calculate flow pressure in the gas channels, and this equation also relates pressure with total gas concentration. This is: 


$$
p_{k}^{j}=R T_{k}^{j} \sum_{i} c_{i, k}^{j}
$$

- Energy balance equations

Accumulation of energy in the gas channels is:

$$
\begin{array}{r}
\frac{d(\rho u)^{j}}{d t}=-\frac{1}{\Delta z}\left(\sum_{i} v^{j} c_{i, k}^{j} h_{i, k}\left(T_{k}^{j}\right)\right) \\
+\frac{1}{\Delta z}\left(\sum_{i} v^{j} c_{i, k-1}^{j} h_{i, k-1}\left(T_{k-1}^{j}\right)\right) \\
+\lambda^{j} \frac{T_{k+1}^{j}-2 T_{k}^{j}+T_{k-1}^{j}}{\Delta z^{2}} \\
+\frac{\alpha_{1}}{\delta^{j}}\left(T_{k}^{S}-T_{k}^{j}\right)-\sum_{i} \frac{\dot{n}_{i, k}^{j}}{\delta^{j}} h_{i, k}\left(T^{j, k}\right)
\end{array}
$$

where energy changes are given by the terms on the right hand side of (6). The first term describes energy transport in the z-direction due to convective flow. The second term represents heat conduction according to Fourier's law [23]. The third term is heat transfer between solid fuel cell parts at temperature $T^{S}$ and gas channels. The last term describes another type of convective flow from gas channels to the MEA. This is also an enthalpy transport.

The boundary equations, using backward differencing are:

$$
\sum_{i} \dot{n}_{i, i n}^{j} h_{i}\left(T_{i n}^{j}\right)=\left.\sum_{i} v_{0}^{j} c_{i, 0}^{j} h_{i}\left(T^{j}\right)\right|_{0, t}-\left.\lambda^{j} \frac{T_{1}^{j}-T_{0}^{j}}{\Delta z}\right|_{0, t}
$$




$$
\left.\lambda^{j} \frac{T_{M Z}^{j}-T_{M Z-1}^{j}}{\Delta z}\right|_{L_{z}, t}=0
$$

- Temperature equations

In order to calculate temperature in the gas channels, a thermodynamic equation of state is used:

$$
(\rho u)_{k}^{j}+p_{k}^{j}=\sum_{i} c_{i}^{j, k} h_{i, k}\left(T_{k}^{j}\right)
$$

\subsubsection{Gas diffusion layers equations}

The purpose of the gas diffusion layers (GDL) model is to introduce a mass transport limitation between gas channels and catalyst layers. The Stefan-Maxwell diffusion equations for a multicomponent species [2] are used to define the gradient in mole fraction of the components. These set of equations is used to calculate mole fractions $\xi_{H_{2}, k}^{C A}$ and $\xi_{O_{2}, k}^{C C}$ in the catalyst layers $(k d$ index is used for mesh points in this case, so there is no confusion with the $k$ index for species in the original equation):

$$
-\nabla \xi_{i, k d}^{j}=\sum_{k} \frac{\bar{\xi}_{k, k d}^{j} \dot{n}_{i, k d}^{j}-\bar{\xi}_{i, k d}^{j} \dot{n}_{k, k d}^{j}}{\bar{c}^{j} D_{i, k}^{e f f}}
$$

The gradient of mole fraction $\nabla \xi_{i}^{j}$ is:

$$
\nabla \xi_{i, k}^{j}=\frac{\xi_{i, k}^{C j}-\xi_{i, k}^{j}}{\delta^{G j}}
$$

The composition inside the GDL $\nabla \xi_{i}^{j}$ is:

$$
\xi_{i}^{j}=\frac{1}{2}\left(\xi_{i, k}^{C j}-\xi_{i, k}^{j}\right)
$$


Finally, the total gas concentration in the GDL follows from:

$$
\bar{c}_{k}^{j}=\frac{p_{k}^{j}}{R T_{k}^{S}}
$$

\subsubsection{Catalyst layers equations}

The catalyst layers equations are used to determine the values of the mass fluxes at each mesh point.

- Mass fluxes through diffusion layers

Due to model assumptions, hydrogen mass flow from the anode gas channel to catalyst layer is identical to the amount of hydrogen consumed in the anodic reaction $\mathrm{H}_{2} \rightarrow 2 \mathrm{H}^{+}+2 \mathrm{e}^{-}$:

$$
\dot{n}_{H_{2}, k}^{A}=r_{k}^{A}
$$

where $r^{A}$ is the rate of the anodic reaction.

The water flow from the anode gas channel to the GDL is passed on to the membrane:

$$
\dot{n}_{H_{2} O, k}^{A}=\dot{n}_{H_{2} O, k}^{A M}
$$

The oxygen transported from the cathode gas channel is completely consumed in the cathodic reaction $\mathrm{O}_{2}+4 \mathrm{e}^{-}+4 \mathrm{H}^{+} \rightarrow 2 \mathrm{H}_{2} \mathrm{O}$. This is:

$$
\dot{n}_{O_{2}, k}^{C}=\frac{1}{2} r_{k}^{C}
$$

Nitrogen flow vanishes, since nitrogen is not a reactant and cannot permeate through the membrane: 


$$
\dot{n}_{N_{2}, k}^{C}=0
$$

Water flow from the cathode is given by the cathode catalyst layer water mass balance:

$$
\dot{n}_{H_{2} O, k}^{C}=\dot{n}_{H_{2} O, k}^{C M}-\frac{1}{2} r_{k}^{C}
$$

- Electrochemistry reactions kinetic rates equations

The reaction rates are modeled by Butler-Volmer equation, which are a current density-potential relations and Faraday's law [20], which states that current density is proportional to the charge transferred and the consumption of reactant per unit area. For anode reaction, this is [19]:

$$
\begin{array}{r}
r^{A}=f^{V} \frac{i_{A 0}}{2 F}\left\{\exp \left(\frac{2 F}{R T_{k}^{S}}\left(\Delta \Phi_{k}^{A}-\Delta \Phi_{r e f}^{A}\right)\right) \frac{\xi_{H_{2}, k}^{C A} p_{k}^{A}}{p_{H_{2}, \text { ref }}}-1\right\} \\
r^{C}=f^{V} \frac{i_{C 0}}{2 F} \exp \left(\frac{\Delta G_{0}}{R}\left(\frac{1}{T_{k}^{S}}-\frac{1}{T_{r e f}}\right)\right) \frac{\xi_{O_{2}, k}^{C C} p_{k}^{C}}{p_{O_{2, r e f}}^{C}} \\
\mathrm{x} \exp \left(\frac{\alpha 2 F}{R T_{k}^{S}}\left(\Delta \Phi_{k}^{C}-\Delta \Phi_{r e f}^{C}\right)\right)
\end{array}
$$

where $\Phi^{A M}$ and $\Phi^{C M}$ are potentials of the membrane on the anode side and on the cathode side.

\subsubsection{Proton exchange membrane (PEM) equations}

The membrane which separates the anode from the cathode must serve several functions. It should efficiently conduct protons, while preventing 
electrons and reactant gasses from crossing, and close the electrical circuit internally by transporting protons from the anode to the cathode. The electrical conductivity of the membrane, strongly depends on membrane humidity. To model this property the simulation model of [18] has been used $(j=A M, C M)$.

- Water concentration equation

Assuming water is only transported along the y-coordinate, perpendicular to gas flow, the water concentration is derived from the following mass balance:

$$
\frac{d c_{H_{2} O}^{M}}{d t}=\frac{1}{\delta^{M}}\left(\dot{n}_{H_{2} O, k}^{A M}+\dot{n}_{H_{2} O, k}^{C M}\right)
$$

- Water content

The protonic conductivity of a polymer membrane is strongly dependent on membrane structure and its water content. The water content $\Lambda$ in membrane is usually expressed as grams of water per gram of polymer dry weight, or as number of water molecules per sulfonic acid groups present in the polymer, $\Lambda=\mathrm{N}\left(\mathrm{H}_{2} \mathrm{O}\right) / \mathrm{N}\left(\mathrm{SO}_{3} \mathrm{H}\right)$. In this case, $\Lambda$ is defined as the ratio between moles of water in the membrane and moles of polymer in the membrane. Water content $\Lambda$ is related to water concentration $c_{\mathrm{H}_{2} \mathrm{O}}^{M}$ by the following relation:

$$
c_{H_{2} O, k}^{M}=\Lambda_{k} \rho_{k}^{M}\left(\Lambda_{k}\right) X_{k}^{M}\left(\Lambda_{k}\right)
$$

Membrane density and ion exchange capacity are, respectively: 


$$
\begin{gathered}
\rho^{M}=\rho_{0} \rho_{d r y} \frac{1+\Lambda_{k} M_{o} X_{d r y}}{\rho_{0}+\Lambda_{k} M_{o} X_{d r y} \rho_{d r y}} \\
X_{M}=\frac{X_{d r y}}{\left(1+\Lambda_{k} M_{o} X_{d r y}\right)}
\end{gathered}
$$

The mole fractions of water protons and protons in the membrane are calculated from:

$$
\begin{aligned}
& \xi_{H_{2} O}\left(\Lambda_{k}\right)=\frac{\Lambda_{k}}{\Lambda_{k}+2} \\
& \xi_{H+}\left(\Lambda_{k}\right)=\frac{1}{\Lambda_{k}+2}
\end{aligned}
$$

- Water fluxes through the membrane equations

The water flows through the membrane are assumed to be driven by gradients of chemical potentials of water and protons. This is an electrochemical method developed by [20], based on electrochemical potential that arises across a membrane sample exposed at each side to different water activities.

$$
\dot{n}_{H_{2} O, k}^{j}=-\frac{t_{W}\left(\Lambda_{k}\right) \kappa\left(\Lambda_{k}\right)}{F^{2}} \nabla \mu_{H+, k}^{j}-\frac{D_{W}\left(\Lambda_{k}\right) c_{H_{2} O, k}^{M}}{R T_{k}^{S}} \nabla \mu_{H_{2} O, k}^{j}
$$

Water is dragged from the anode to the cathode by protons moving through the membrane. This is called electroosmotic drag. The flux of water due to electroosmotic drag is given by the left term of the right hand side of 27. Moreover, water generation and electroosmotic drag create a large 
concentration gradient across the membrane. Because of this gradient, water diffuses back from the cathode to the anode. The other term of the right hand side reflects back-diffusion of water. The electroosmotic drag coefficient $t_{W}$ relation and the water diffusion coefficient $D_{W}$ relation are taken from [18].

The gradients of chemical potential are calculated from:

$$
\begin{gathered}
\nabla \mu_{H_{2} O, k}^{j}=\frac{R T^{S}, k}{\xi_{H_{2} O, k}(\Lambda)} \nabla \xi_{H_{2} O, k}^{j} \\
\nabla \mu_{H+, k}^{j}=\frac{R T^{S}}{\xi_{H+, k}\left(\Lambda_{k}\right)} \nabla \xi_{H+, k}^{j}+F \nabla \Phi \\
\nabla \xi_{H 2_{O}}^{j}=\frac{\xi_{H_{2} O}(\Lambda)-\xi_{H_{2} O}\left(\Lambda^{j}\right)}{\delta^{M} / 2}
\end{gathered}
$$

As it was done in the GDLs model, the gradients are approximated by simple difference formulas:

$$
\begin{gathered}
\nabla \xi_{H+}^{j}=\frac{\xi_{H+}(\Lambda)-\xi_{H+}\left(\Lambda^{j}\right)}{\delta^{M} / 2} \\
\nabla \Phi=\frac{\Phi^{C} M-\Phi^{A} M}{\delta^{M}}
\end{gathered}
$$

Water contents at membrane boundaries to the anode side $\Lambda^{A M}$ and cathode side $\Lambda^{C M}$ depend on the relative humidity in the catalyst layers. As shown in the next chapter, calculation is given by sorption isotherms taken from $[18]$.

- Electrical current density equations 
Electrical current through the membrane is related to proton flow by:

$$
i^{M}=F \dot{n}_{H+, k}
$$

- Proton flux equations

Similar to the water flux, the proton flux is driven by gradients of electrochemical potentials:

$$
\dot{n}_{H+, k}=-\frac{\kappa\left(\Lambda_{k}\right)}{F^{2}} \nabla \mu_{H+}-\frac{t_{W}\left(\Lambda_{k}\right) \kappa\left(\Lambda_{k}\right)}{F^{2}} \nabla \mu_{H_{2} O}
$$

Considering there is no accumulation of protons in the membrane, is calculated as follows. This is a set of equations added to the [6] simulation model.

$$
\begin{gathered}
\nabla \mu_{H_{2} O, k}=\frac{R T_{k}^{S}}{\xi_{H_{2} O, k}\left(\Lambda_{k}\right)} \nabla \xi_{H_{2} O} \\
\nabla \mu_{H+, k}=\frac{R T^{S}}{\xi_{H+, k}\left(\Lambda_{k}\right)} \nabla \xi_{H+}+F \nabla \Phi \\
\nabla \xi_{H 2_{O}, k}=\frac{\xi_{H_{2} O, k}\left(\Lambda_{k}^{C M}\right)-\xi_{H_{2} O, k}\left(\Lambda_{k}^{A M}\right)}{\delta^{M}} \\
\nabla \xi_{H+, k}^{j}=\frac{\xi_{H+}\left(\Lambda_{k}^{C M}\right)-\xi_{H+, k}\left(\Lambda^{A M}\right)}{\delta^{M}} \\
\nabla \Phi_{k}=\frac{\Phi^{C} M_{k}-\Phi^{A} M_{k}}{\delta^{M}}
\end{gathered}
$$




\subsubsection{Solid part energy balance}

Similar to the gas channels energy balance, the energy balance for the solid part ( $\mathrm{SP})$ is:

$$
\begin{array}{r}
\delta^{s} \frac{\partial(\rho e)^{j}}{\partial t}=\sum_{i, j} \dot{n}_{i}^{j} h_{i}\left(T_{k}^{j}\right)+\sum_{j=A, C} \alpha_{1}\left(T_{k}^{j}-T_{k}^{S}\right) \\
+\alpha_{2}\left(T_{k}^{c o o l}-T_{k}^{S}\right)+ \\
\lambda^{s} \delta^{s} \frac{T^{j}}{\Delta z^{2}}-\left(\Phi_{k}^{C}-\Phi_{k}^{A}\right) i_{k}^{M}
\end{array}
$$

where energy changes are given by the terms on the right hand side of 40 . This is mass exchange between gas channels and SP, heat exchange between gas channels and SP, heat exchange between coolant and SP, Fourier heat conduction and electrical work.

The total energy relation is defined as internal energy (enthalpies of the different parts of the MEA) and electrical energy in the double layers. This is:

$$
\begin{array}{r}
\delta^{s}\left(\rho e_{k}\right)=\delta^{s}\left(\rho u_{k}\right)+C^{A} \delta_{k}^{A C} \frac{\Delta \Phi_{k}^{A^{2}}}{2}+C^{C} \delta_{k}^{C C} \frac{\Delta \Phi_{k}^{C^{2}}}{2} \\
=\delta^{s}(\overline{\rho h})\left(T^{S}\right)+C^{A} \delta^{A C} \frac{\Delta \Phi^{A^{2}}}{2}+C^{C} \delta^{C C} \frac{\Delta \Phi^{C^{2}}}{2}
\end{array}
$$

where: 


$$
\begin{gathered}
\delta^{s}(\overline{\rho h})\left(T_{k}^{S}\right)=\left(\delta^{S}-\delta^{M}\right)(\rho h)_{k}^{S}\left(T_{k}^{S}\right)+ \\
\delta^{M}\left(\rho h_{k}\right)^{M}\left(T_{k}^{S}\right)+\delta^{M} \rho_{H_{2} O, k}^{M} h_{H_{2} O, k}\left(T_{k}^{S}\right)
\end{gathered}
$$

\subsubsection{Conservation of charge equations}

Current transport is described by a governing equations for conservation

of charge [20]. In this model, it is assumed that charge is transported in the direction of the z-coordinate, and through the membrane, in the direction of the y-coordinate. Charge balances are:

$$
\begin{gathered}
C^{A} \delta^{A C} \frac{d \Delta \Phi_{k}^{A}}{d t}=i_{k}^{M}-2 F r_{k}^{A} \\
C^{C} \delta^{C C} \frac{d \Delta \Phi^{C}}{d t}=i_{k}^{M}-2 F r_{k}^{C}
\end{gathered}
$$

\subsubsection{Cell current}

The total cell current is given by [20]:

$$
I=L_{x}\left(\sum_{k}^{L_{z}} i_{k}^{M}\right) \Delta z
$$

\subsubsection{Cell potential}

$$
U(t)=\Delta \Phi^{C}(z, t)-\Delta \Phi^{M}(z, t)-\Delta \Phi^{A}(z, t)
$$

In this relation activation polarization losses (energy activation barrier) and ohmic losses (potential drop in the membrane) are considered. 


\subsection{Numerical implementation}

The simulation model consists of six submodels that are coupled by internal variables. The GC module models reactant concentrations, flow velocity, pressure and temperature in the gas channels. The GDL module studies diffusion in a multicomponent mix of species. The electrochemical reactions and mass fluxes are modeled in the CL submodel. The membrane module consists of a detailed protonic exchange membrane model [8].

The solid part module consists of an energy balance to determine the solid part temperature. Finally, cell current and voltage are determined in the charge balances module. The inputs are inlet flows for anode side and cathode side, inlet flows temperatures, cooling temperature and cell voltage. The main output is cell current, but it is possible to have cell current as an input instead of cell voltage. This depends on the operation mode desired.

The set of model equations (DAE) was implemented and numerically solved with Matlabß) using the ODE15s solver (DAE solver). Performing simulations is very simple. There are two main m-files, the model equations file and the model simulation file. The former is a function file that contains the whole set of differential algebraic equations (all submodels equations coupled by each module variables), the latter generates the numerical solutions, by calling the solver, and the graphics of variables dynamics (set of surfaces).

There are more options to generate specific graphics such as along-thechannel steady-state results, along-time results and specific outputs such as oxygen and hydrogen stoichiometry, relative humidity, and others, but these are optional m-files. It is also possible to use each submodule separately (separate m-files), by considering coupling variables as constant profiles. This 
option is suitable when studying the behavior of specific cell components.

The tool is intended to analyze along-the-channel behavior through time, and design model-based controllers that consider spatial variations. For this purpose the model has to be reduced by means of complex model order reduction techniques. However, as ongoing work, the simulation tool is being migrated to Simulink, to precisely make it suitable to a larger system simulation and test conventional controllers such as FeedForward or PID. This means using the tool as a lumped parameter model, and still being able to study spatial profiles of PEMFC variables.

\section{Simulation results}

In order to show the possibilities of the tool, the results of simulations for different variables are shown. The inputs to the model are: hydrogen and water inflow on anode side, oxygen, water and nitrogen inflow on cathode side, temperature of inflows, cell voltage and coolant temperature. Operation conditions are, respectively: $\dot{n}_{H_{2}}^{A}=10 \mathrm{~mol} \mathrm{~m}^{-2} \mathrm{~s}^{-1}, \dot{n}_{H_{2} O}^{A}=0.5 \mathrm{~mol} \mathrm{~m}^{-2} \mathrm{~s}^{-1}$, $\dot{n}_{\mathrm{O}_{2}}^{C}=10 \mathrm{~mol} \mathrm{~m} \mathrm{~m}^{-2} \mathrm{~s}^{-1}, \dot{n}_{H_{2} O}^{C}=7 \mathrm{~mol} \mathrm{~m}^{-2} \mathrm{~s}^{-1}, \dot{n}_{N_{2}}^{C}=35 \mathrm{~mol} \mathrm{~m}^{-2} \mathrm{~s}^{-1}, T^{A}=$

$T^{C}=353 \mathrm{~K}$, Volt $=0.8 \mathrm{~V}$ and $\mathrm{Tcool}=345 \mathrm{~K}$. The number of mesh points is $M Z=11$. Some important model parameters are: $L_{z}=0.4 \mathrm{~m}, L_{x}=10^{-3} \mathrm{~m}$ and $\alpha_{1}=100 \mathrm{Wm}^{-2} \mathrm{~K}^{-1}$.

\subsection{Steady-state simulation results}

The steady-state results of some important variables considered in the model are presented in this section. Fig. 2 shows spatial profiles of the concentration of reactants (hydrogen and oxygen), nitrogen and water in anode and cathode gas channels. Reactants concentrations are higher at the 
beginning of the channels, which is the zone of major exothermic reaction rate. In this pressure driven model, nitrogen concentration changes because of flow velocity variation along the channel. Water increases in both sides of the membrane due to water generation on cathode side and back-diffusion on anode side.

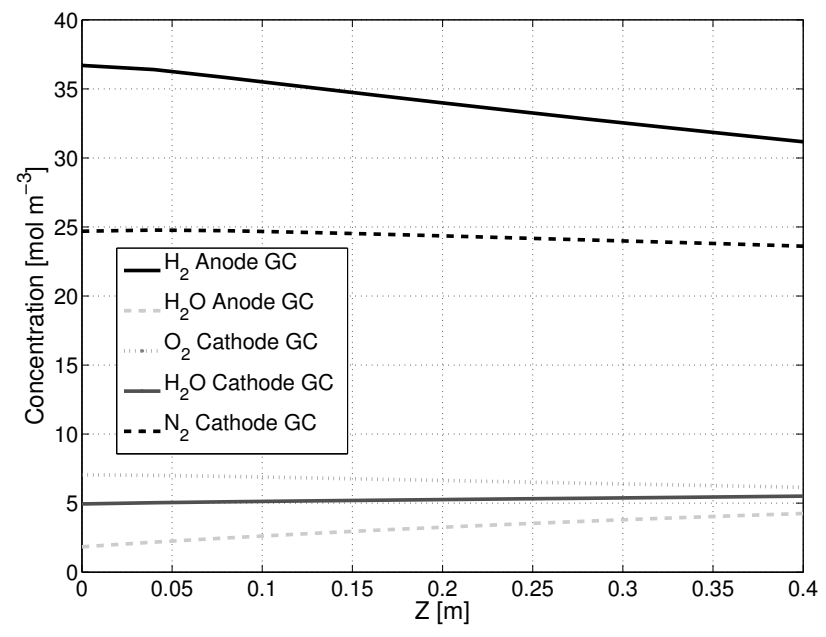

Figure 2: Spatial profiles of concentrations of reactants, nitrogen and water in the gas channels.

Heterogeneities of the reactants concentrations observed along the channels (Fig. 2) are of major importance for studying and understanding degradation phenomena such as carbon corrosion [24, 25, 26], which is highly enhanced by fuel starvation in the anode.

Fig. 3 shows the current density profile along the channel (right side). It can be seen that the current (and the reaction rate) is higher close to entrance of gasses, where the reactants have higher concentrations. This figure also shows spatial profiles of the different temperature levels. As can be seen, temperature is higher at the zone of major exothermic reaction rate and the 
temperature of the gas channels follows the SP temperature.

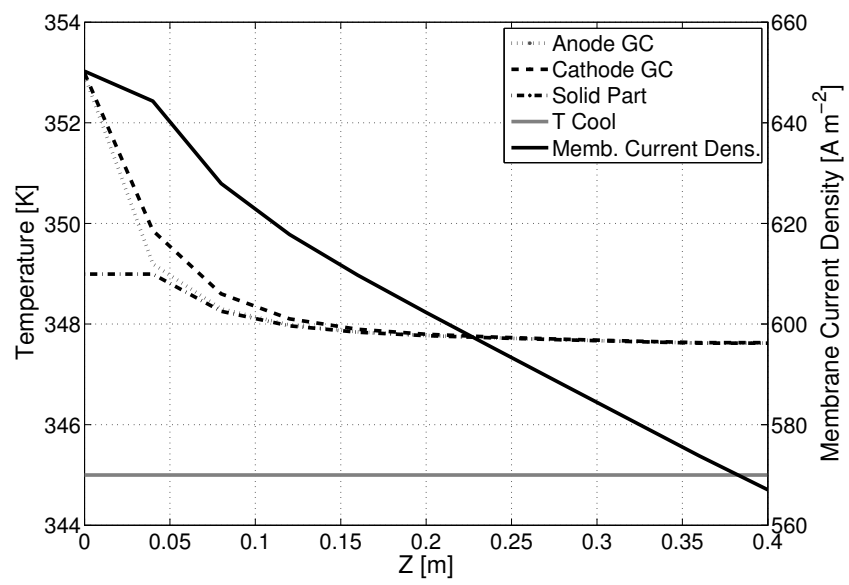

Figure 3: Spatial profiles of membrane current density and temperature in the gas channels, solid part and cooling level.

Pressure variations cause nitrogen concentration to decrease but nitrogen flow rate (not shown) is actually constant (35 mol m-2 s-1), because nitrogen is not a reactant. Due to boundary conditions and a relatively small number of mesh points, spatial profiles close to the inlet of gas channel are not very smooth.

Fig. 4 shows spatial profiles for membrane water content, together with spatial profiles of water transport from membrane to anode and cathode catalyst layers.

As expected, membrane water content is higher at the end of the $\mathrm{z}$ direction and water is transported from both sides of membrane to the catalyst layers due to water generated, electroosmotic drag and water diffusion. This agrees with the results shown in Fig. 2, where water concentration increases towards the end of the channel on both sides of the membrane. 


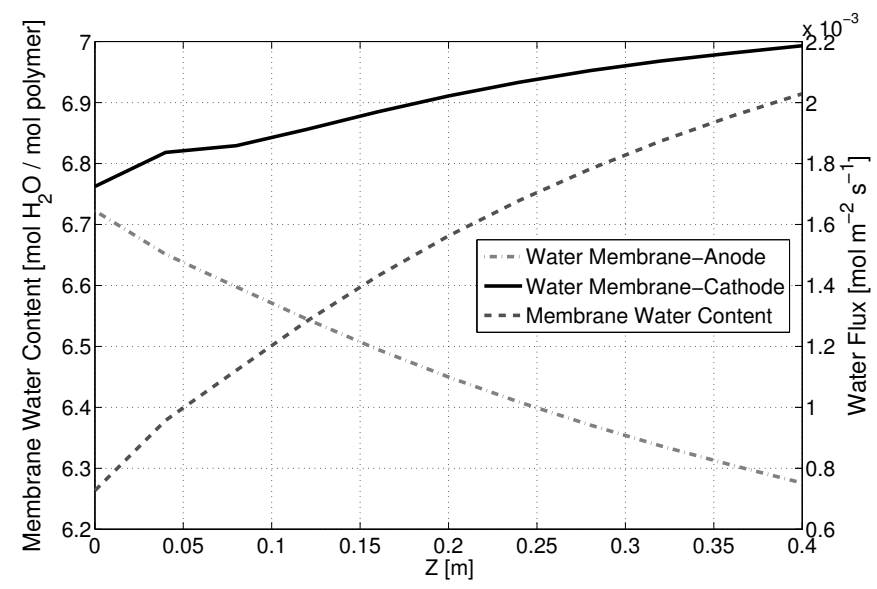

Figure 4: Spatial profiles of membrane water content, water transport from membrane to anode and cathode catalyst catalyst layers.

Finally, Fig. 5 shows a polarization curve obtained from the simulation tool, in order to show its capacity to study experimental observables. Normal values for PEMFC operation result for the range of operating conditions considered in this $\mathrm{i}-\mathrm{V}$ example.

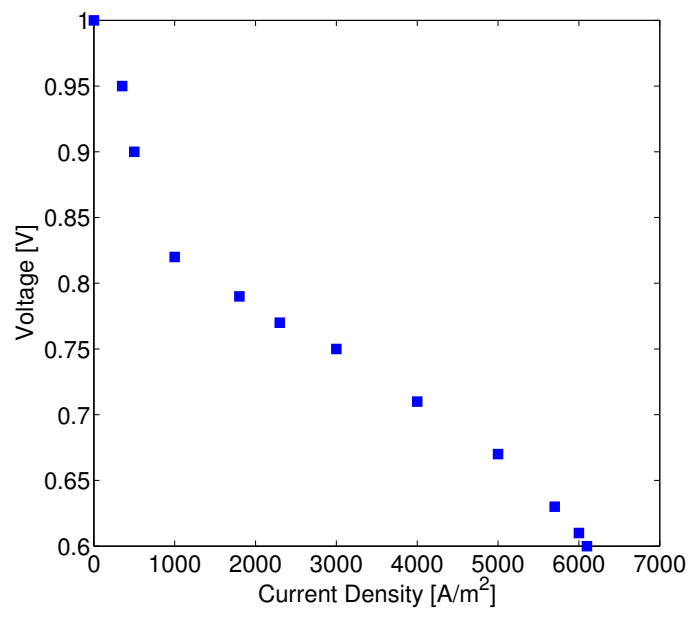

Figure 5: Polarization curve 


\subsection{Simulation results for step changes}

At time $\mathrm{t}=10 \mathrm{~s}$ the inflow of oxygen is increased up to $14 \mathrm{~mol} \mathrm{~m}^{-2} \mathrm{~s}^{-1}$ for $100 \mathrm{~s}$, then at time $\mathrm{t}=210 \mathrm{~s}$, inflow of hydrogen is changed up to 12 mol m $\mathrm{m}^{-2} \mathrm{~s}^{-1}$ for $100 \mathrm{~s}$ and finally, water inflow in the cathode is changed down to $6 \mathrm{~mol} \mathrm{~m}^{-2} \mathrm{~s}^{-1}$. Fig. 6 shows the results of time variation of reactant and water concentrations for the middle point of the channel.

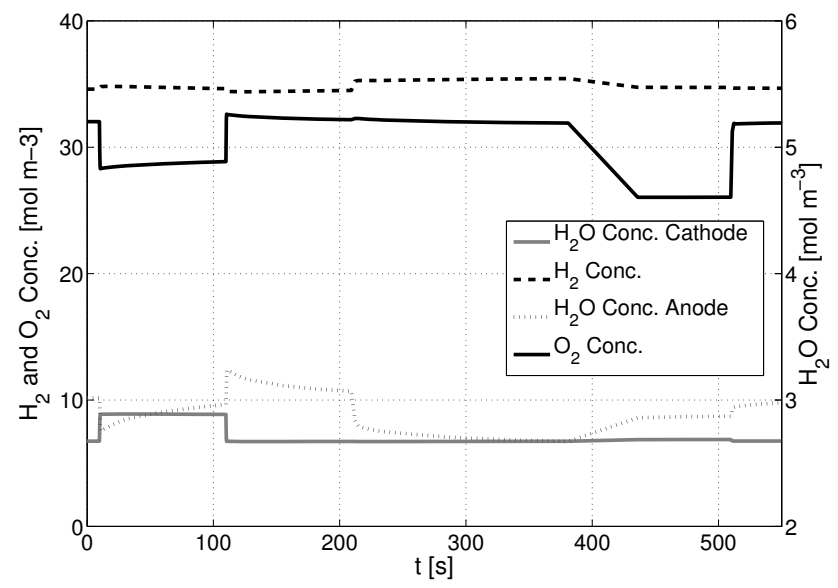

Figure 6: Time evolution of reactant and water concentration at the middle point of the channel.

Fig. 7 shows the same type of diagram for membrane current density and membrane water content, but considering three points along the channel. The increase in reactants concentrations effectively increases membrane current density and water content. It is important to notice the different response of the system depending on the channel point. Changes at the first point of the channel are almost immediate, whereas there is a slower time constant further along the channel. 


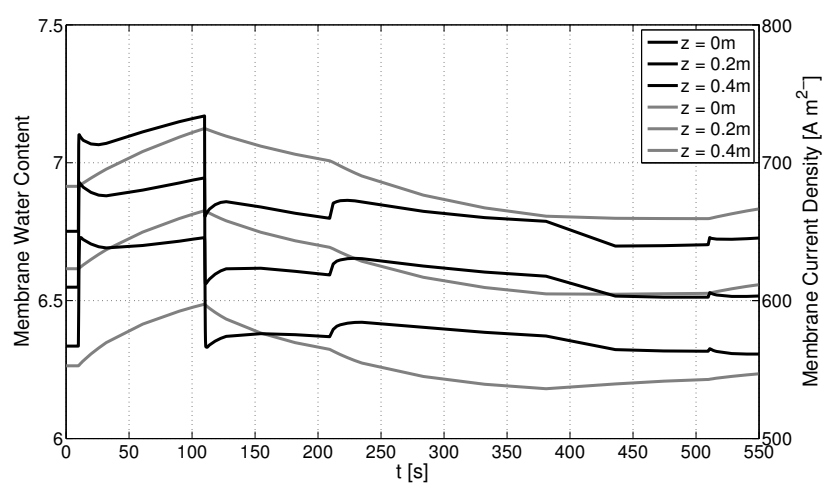

Figure 7: Time evolution of membrane water content and current density at the beginning point, middle point and channel end. Black lines correspond to membrane current density.

\section{Conclusions}

A two-dimensional PEMFC simulation tool, suitable for along-the-channel studies, has been developed in Matlabß. The tool is flexible enough to apply order reduction techniques and to be used in model-based control design. Simulation results show the importance of considering, and therefore, controlling aspects of spatial profiles, especially for PEMFC performance and durability issues. It is the intention of the authors to make the source code publicly available.

\section{Acknowledgements}

This work has been supported by national projects DISCPICO with reference DPI2010-15274, MESPEM with reference DPI2011-25649 and european project PUMA MIND. 


\section{References}

[1] I. S. A. I. C. Parsons, Fuel Cell Handbook, U.S. Department of Energy, 2000.

[2] D. Bernardi, M. Verbrugge, Mathematical model of a gas diffusion electrode bonded to polymer electrolyte, AIChE 37 (1991).

[3] C. Siegel, Review of computational heat and mass transfer modeling in polymer-electrolyte-membrane (PEM) fuel cells, Energy 33 (2008) $1331-1352$.

[4] A. Rowe, X. Li, Mathematical modeling of proton exchange membrane fuel cells, Journal of Power Sources 102 (2001) 82-96.

[5] R. Methekar, V. Prasad, R. Gudi, Dynamic analysis and linear control strategies for proton exchange membrane fuel cell using a distributed parameter model, Journal of Power Sources 165 (2007) 152-170.

[6] M. Mangold, A. Bück, R. Hanke-Rauschenbach, Passivity based control of a distributed pem fuel cell model, Journal of Process Control 20 (2010) 292-313.

[7] S. Um, C. Y. Wang, K. S. Chen, Computational fluid dynamics modeling of proton exchange membrane fuel cells, Journal of The Electrochemical Society 147 (2000) 4485-4493.

[8] A. Kulikovsky, Semi-analytical 1D + 1D model of a polymer electrolyte fuel cell, Electrochemistry Communications 6 (2004) 969 - 977. 
[9] M. H. Nehrir, C. Wang, Modeling and Control of Fuel Cells: Distributed Generation Applications, Wiley-IEEE Press, 2009.

[10] J. Schumacher, J. Eller, G. Sartoris, A $2+1 \mathrm{D}$ model of a proton exchange membrane fuel cell with glassy-carbon micro-structures, in: Proceedings MATHMOD, pp. 16-19.

[11] W. Tao, C. Min, X. Liu, Y. He, B. Yin, W. Jiang, Parameter sensitivity examination and discussion of pem fuel cell simulation model validation: Part i. current status of modeling research and model development, Journal of Power Sources 160 (2006) 359-373.

[12] S. Um, C. Wang, Three-dimensional analysis of transport and electrochemical reactions in polymer electrolyte fuel cells, Journal of Power Sources 125 (2004) $40-51$.

[13] A. A. Franco, A multiscale modeling framework for the transient analysis of PEM Fuel Cells - From the fundamentals to the engineering practice., Université Claude Bernard Lyon 1, 2010.

[14] R. F. de Morais, P. Sautet, D. Loffreda, A. A. Franco, A multiscale theoretical methodology for the calculation of electrochemical observables from ab initio data: Application to the oxygen reduction reaction in a Pt(1 1 1)-based polymer electrolyte membrane fuel cell, Electrochimica Acta 56 (2011) $10842-10856$.

[15] A. A. Franco, P. Schott, C. Jallut, B. Maschke, A multi-scale dynamic mechanistic model for the transient analysis of PEFCs, Fuel Cells 7 (2007) 99-117. 
[16] COMSOL, Comsol, http://www.comsol.com/, 2012.

[17] E. Verriest, Time variant balancing and nonlinear balanced realizations, in: W. H. A. Schilders, H. A. van der Vorst, J. Rommes (Eds.), Model order reduction. Theory, research aspects and applications, Springer, 2008.

[18] W. Neubrand, Modellbildung und Sumulation von Elektromembranverfahren, Logos-Verlag, 1999.

[19] M. Wöhr, Instationäres, thermodynamisches Verhalten der Polymermembra-Brennstoffzelle, VDI-Verlag, 1999.

[20] F. Barbir, PEM Fuel Cells: Theory and Practice, Academic Press, 2005.

[21] J. Sjöberg, Optimal control and model reduction of nonlinear DAE models, Ph.D. thesis, Linköping University, Sweden, 2008.

[22] P. K. Kundu, I. M. Cohen, Fluid Mechanics, Academic Press, 1990.

[23] H. D. B. Jenkins, Chemical Thermodynamics at a Glance, Blackwell Publishing, 2008.

[24] K. Malek, A. A. Franco, Microstructure-based modeling of aging mechanisms in catalyst layers of polymer electrolyte fuel cells, The Journal of Physical Chemistry B 115 (2011) 8088-8101.

[25] A. A. Franco, M. Gerard, Transient model of carbon catalyst-support corrosion in a PEFC: Multi-scale coupling with Pt electro-catalysis and impact on performance degradation, Meeting Abstracts MA2008-01 (2008) 1160. 
[26] J. Wu, X. Yuan, J. Martin, H. Wang, J. Zhang, J. Shen, S. Wu, W. Merida, A review of pem fuel cell durability: Degradation mechanisms and mitigation strategies, Journal of Power Sources 184 (2008) 104-119. 